\title{
Análisis de los procesos logísticos de una organización mediante el modelo Donald Bowersox
}

\section{Analysis of an organization's logistics processes using the Donald Bowersox model}

Harold Steve Lora-Guzmán

Universidad de Cartagena - Colombia

ORCID iD: https://orcid.org/0000-0001-6945-0999

hlorag@unicartagena.edu.co

\section{Luis Guillermo Garcés-Henao*}

Escuela Naval de Cadetes "Almirante Padilla" -

Colombia

ORCID iD: https://orcid.org/0000-0002-0733-6055

lggarcesh@enap.edu.co

* Autor a quien debe ser dirigida la correspondencia
Fecha de recepción: 08/10/2019

Fecha de evaluación: 06/02/2020

Fecha de aceptación: 10/05/2020

Cómo citar: Lora-Guzmán, H., \& Garcés-Henao, L. (2020). Análisis de los procesos logísticos de una organización mediante el modelo Donald Bowersox. Revista Cientifica Anfibios, 3(1), 52-59. https://doi.org/10.37979/afb.2020v3n1.62

\section{Resumen}

En la actualidad con fenómenos como la globalización y el progreso de las tecnologías de la información y comunicación, la gestión logística se considera como uno de las alternativas más importantes para mejorar el desempeño de las organizaciones, su competitividad y la satisfacción de los clientes (Companys \& Ribas, 2015). Bajo este marco, señalar las razones por las cuales los consumidores no se encuentran satisfechos con los productos o servicios recibidos puede estar relacionado con demoras en la entrega, precios altos o productos y servicios de mala calidad, cuyo fundamento recae en la cadena logística. Es por estos motivos que se hace necesario analizar dichos procesos en búsqueda de estas falencias que permitan mejorar los resultados y generar competitividad. Este es el caso de una organización que muestra algunas dificultades en atención y servicio a sus clientes, es por esto que mediante el paradigma del Modelo Referencial Logístico de Donald Bowersox, se localizan estas dificultades, así como mecanismos para resolverlas. Los resultados principales muestran un diagnostico organizacional en la que 9 de las 13 dimensiones planteadas por Bowersox presentan brechas considerables y una posibilidad de mejora y a partir de allí, se plantean conclusiones y discusiones en las que se pueden orientar futuros trabajos investigativos.

\section{Palabras claves:}

Logística; Modelo referencial logístico; Gestión; Competitividad; Desempeño de las organizaciones

\begin{abstract}
Currently, with phenomena such as globalization and the progress of information and communication technologies, logistics management is considered one of the most important alternatives for improving the performance of organizations, their competitiveness and customer satisfaction (Companys \& $\mathrm{Ri}$ bas, 2015). Under this framework, pointing out the reasons why consumers are not satisfied with the products or services received can be related to delays in delivery, high prices or poor quality products and services, the basis of which lies in the logistics chain. It is for these reasons that it is necessary to analyse these processes in search of these shortcomings in order to improve results and generate competitiveness. This is the case of an organisation that shows some difficulties in attention and service to
\end{abstract}


its clients, which is why through the paradigm of the Donald Bowersox Logistical Reference Model, these difficulties are located, as well as mechanisms to solve them. The main results show an organizational diagnosis in which 9 of the 13 dimensions proposed by Bowersox present considerable gaps and a possibility of improvement, and from there, conclusions and discussions are proposed in which future research work can be oriented.

\section{Keywords}

Logistics; Logistics reference model; Management; Competitiveness; Organisational performance

\section{Introducción}

En la actualidad, aquellas organizaciones que no tengan el control de sus procesos productivos y logísticos enfocados hacia sus clientes o consumidores no está en la posición de competir (Del Río Cortina et al., 2009), por lo tanto, son importantes estos procesos para un correcto funcionamiento de toda la cadena productiva y posibilitar la detección de los obstáculos y errores del mismo (Hernández et al., 2017)es establecer el nivel de innovación en tecnología y direccionamiento estratégico de las pequeñas y medianas empresas (Pymes.

Para mejorar la competitividad y asegurar los objetivos institucionales de la organización es necesario analizar sus procesos y sistemas logísticos para establecer una comprensión amplia y clara con el fin de desarrollar estrategias para que aquellas áreas que requieren intervención o tengan susceptibilidad de mejoras permitan el desarrollo de planes, decisiones y lineamientos para para incrementar la competitividad.

Es así como una organización que realiza sus procesos de acuerdo con la normativa administrativa vigente y las actividades necesarias para cumplir la misión de proporcionar servicios a sus clientes, no obstante, es posible que se puedan presentar errores u obstáculos debido a malentendidos o errores en los procedimientos administrativos y logísticos, lo que complica el desarrollo normal de todos sus ámbitos organizacionales dado que no existe una visión holística y actualizada del estado de su personal y su dependencia del conocimiento logístico y los métodos de organización para el manejo, almacenamiento, suministro y materiales de alimentos, transporte interno y externo, sistemas de comunicación, software empresarial, talento, integración de la cadena de suministro, logística inversa y medición del desempeño logístico.

En ese orden de ideas, partiendo de un apartado teórico en donde se vincula el área logística de una organización con su competitividad, además de las características que presentan a nivel logístico las organizaciones inmersas en el sector servicios, se plantean las brechas como línea base en la toma de decisiones para la identificación de oportunidades de mejora en los procesos de acuerdo a las dimensiones previamente mencionadas y propuestas por el modelo referencial logístico de Donald Bowersox, posibilitando el diagnostico de las dificultades y obstáculos a nivel logístico y finalmente plantear algunas discusiones sobre futuros trabajos que permitan aumenta la competitividad de la organización y de sus procesos logísticos.

\section{Apartado Teórico}

\section{La competitividad desde el sector logístico}

Para Porter (2015) el éxito o fracaso de una organización depende del correcto desarrollo de sus actividades sumado a innovaciones, cohesión entre los diferentes departamentos y una adecuada implementación de estrategias, esto asegurándose que la organización alcance una posición rentable y sostenible tomando en consideración las fuerzas que rigen el sector de la misma, ofrecen una definición de competitividad. En ese sentido, Gutiérrez (2010) define a una organización competitiva cuando esta es capaz de generar productos y servicios mejores que la competencia. 
Bajo este marco, las organizaciones deben estar en capacidad de identificar las ventajas que se poseen frente a la competencia y de esa manera hacer uso de estrategias favorables para el negocio (Durán et al., 2016). Es así como la ventaja competitiva se logra proporcionando valor a los clientes por medio de la disminución de costos y el aumento del desempeño de la organización y para esto es necesario que los procesos logístico organizacionales se lleven a una correcta ejecución tomando en cuenta factores como costos, calidad, respuesta oportuna y flexible, conduciendo a la rentabilidad de la organización (Presutti, 2013).

Es por lo anterior que resulta importante la Gestión de la Cadena de Suministro la cual comprende todas las actividades de planificación, compras, fabricación, logística de entrada, logística interna, logística de salida y logística inversa, determinando la coordinación de las funciones de las áreas organizacionales para lograr la satisfacción de los clientes internos y externos, los accionistas y toda la comunidad (Prieto et al., 2016).

Por su parte, la gestión de la cadena de abastecimiento es una estrategia global que se encarga de administrar conjuntamente las funciones, procesos, actividades y agentes que conforman la cadena de suministro, y busca que cada función, proceso, actividad o empresa ya no opere de manera aislada para lograr sus objetivos sino constituirla como un proyecto conjunto y constituir la cadena de suministro como un proyecto. Deje que todo el equipo avance hacia el objetivo común (Adarme-Jaimes et al., 2012).

\section{El Modelo Logístico en el sector servicios}

El objetivo principal de un modelo logístico es establecer el nivel óptimo de volumen o cantidades de pedidos que permita minimizar los costes totales de la gestión de inventario (Salas-Navarro et al., 2019), sin embargo, el sector servicio ofrece una estructura diferente al sector comercial e industrial, por lo tanto, requiere su propio análisis en modelos logísticos.

Rojas De Francisco et al., (2016) destacan cinco modelos de gestión logística para organi- zaciones dedicadas al sector servicio, estas son; La Separación total entre Productos y Servicios; Unidades Estratégicas de Negocio; Desarrollo Nuevos Servicios; Triada de Servicio; Cocreación; y finalmente funciones de servicios, sin embargo, aunque cada uno cuenta con sus características, en el presente artículo el modelo referencial logístico utilizado será de Donald Bowersox, el cual cumple con las características requeridas para los objetivos planteados.

Una herramienta que permite la recopilación de conceptos holísticos que podría aplicarse a la Gestión de la Cadena de Suministro de organizaciones del sector servicio, es el Modelo Referencial Logístico (MRL) de Donald Bowersox que tiene por objetivo además de alcanzar la competitividad de la organización, también persigue el mejoramiento de toda la cadena de suministro y de los sistemas logísticos de la misma. Dicho modelo consta de 13 factores como son; Concepto sobre logística; Organización logística; Tecnología de la manipulación; Tecnología de almacenaje; Tecnología de transporte interno; Tecnología de transporte externo; Tecnología de comunicaciones; Tecnología de software; Talento humano; Integración de Supply Chain; Barreras logísticas; Logística reversa; y Medida de desempeño logístico.

Estos factores permiten el monitoreo y la planificación de estrategias operativas, que se reflejan en los objetivos propuestos implementados a través del plan de acción, además, deben ser; simples y claros, esto es que se pueden identificar rápidamente información clave; Fáciles de preparar rápidamente para garantizar una liberación regular; Relevantes es decir, que contiene datos relacionados con la actividad de investigación y finalmente adaptarse a cada función, industria, proyecto o cada necesidad.

Por estos motivos se ha determinado para el presente artículo realizar la medición de los procesos logísticos y administrativos de una organización modelo Donald Bowersox.

\section{Metodología}

El Modelo Referencial Logístico de Bowersox et al., (1986) pretende identificar los factores 
determinantes de éxito internos y externos, así como otros factores que inciden en el desarrollo de los sistemas logísticos de un organización, describiendo sus características con el fin de elaborar un diagnóstico que le permita a la organización implementar herramientas para el mejoramiento de su gestión logística. Para tales fines se llevar a cabo un diagnostico mediante el Modelo Referencial Logístico propuesto obtener información necesaria para los análisis correspondientes.

Este modelo se lleva a cabo por medio de cuatro etapas. La primera hace referencia a la revisión, definición y documentación de las actividades y procesos logísticos al interior de la organización, con la finalidad de recabar información preliminar que de soporte a las siguientes etapas. Seguidamente se establecen las técnicas adecuadas para el diagnóstico y evaluación del sistema logístico de la organización, estableciendo indicadores en base a las dimensiones anteriormente mencionadas a continuación.

Este diagnóstico se hace en base a dos cálculos, en primera instancia la variable resultado permite medir de 1 a 5 , en donde 1 es ineficiencia y 5 es eficientice, en ese orden de ideas el modelo permita calcular la brecha o GAP que corresponde a la siguiente ecuación:

$\mathrm{G}(\mathrm{GAP})=5.0$ (condiciones ideales) $-\mathrm{R}$ (resultado de la medición)

En la cual, el puntaje de 5.0 indica condiciones ideales y el puntaje obtenido en cada una de las tecnologías. En base a esto las calificaciones son; Excelente (de 5,0 a 4,0); Bueno $(3,0$ a 4,9); Insuficiente (de 2,9 a 2,0$)$ y deficiente (de 1,9 a 0$)$.

Posteriormente se compara y analizan la información obtenida y se contrasta con los indicadores en las dimensiones establecidas por el modelo de Bowersox y finalmente se plantean estrategias de mejora y lineamientos que permitan mejorar el proceso logístico al inte- rior de la organización tomando como base las dificultades encontradas.

\section{Hallazgos}

Los resultados preliminares del modelo Referencial logístico arrojaron inconvenientes en las siguientes categorías. Desde el punto de vista de adquisiciones y el primer eslabón de toda la cadena de suministros de la organización se identificó que existe una muy baja planificación respecto a los insumos y materias primas o servicios necesarios para la operación, esto evidentemente afecta la atención en la medida que no se presenta una cotización y selección previa que permita elegir los mejores proveedores terminando esto en un incremento en los costos debido a la inmediatez. En este mismo sentido, también se pudo constatar la falta de especificación en materiales y servicios, debido a que no se tiene una planificación previa de los insumos operacionales, tampoco se especifica exactamente los materiales que se necesitan, es por esto que hay presencia de productos no deseados en los almacenes además de procesos de recompra que son costosos e ineficientes.

En el apartado de inventario y abastecimiento, este presenta inconvenientes en la baja rotación de almacenes, haciendo que muchos productos estén caducados además de la presencia de insumos no deseados debido a que no cumplen con las características específicas necesarias para ser utilizados en el proceso productivo. Asimismo, no se cuenta con un sistema que permita conocer en tiempo real cuales son las existencias de insumos y productos necesarios para la operación, haciendo que se improvise al momento de ordenar compras y presentando una gran ineficiencia.

En cuanto la operación de la organización, las áreas funcionales presentan ineficiencias y demoras en la atención del cliente hecho que dificulta un buen servicio. Tomando en cuenta todo lo anterior, los resultados desde el paradigma del Modelo Referencial Logístico se pueden observar en la siguiente tabla. 
Tabla 1. Brechas detectadas por el Modelo Referencial Logístico

\begin{tabular}{lrrrr}
\hline \multicolumn{1}{c}{ Tecnología } & MRL & Resultado & Gap & Calificación \\
Barreras del entorno & 5 & 2,81 & 2,19 & Insuficiente \\
Concepto logístico & 5 & 3,30 & 1,70 & Bueno \\
Integración del Supply Chain & 5 & 2.38 & 2,62 & Insuficiente \\
Logística de reversa & 5 & 3,42 & 1,58 & Bueno \\
Medida del desempeño logístico & 5 & 2,34 & 2,66 & Insuficiente \\
Organización y gestión logística & 5 & 2,92 & 2,08 & Insuficiente \\
Talento humano & 5 & 3,17 & 1,83 & Bueno \\
Tecnología de almacenaje & 5 & 2,92 & 2,08 & Insuficiente \\
Tecnología de información & 5 & 2,69 & 2,31 & Insuficiente \\
Tecnología de manipulación & 5 & 1,28 & 1,72 & Bueno \\
Tecnología de software & 5 & 2,50 & 2,50 & Insuficiente \\
Tecnología de transporte externo & 5 & 2,83 & 2,17 & Insuficiente \\
Tecnología de transporte interno & 5 & 2,86 & 2,14 & Insuficiente \\
Promedio general del modelo & 5 & 2,88 & 2,12 & Insuficiente \\
\hline
\end{tabular}

Fuente: Elaboración propia.

De acuerdo con los resultados, existen muchas oportunidades de mejora al interior de cada categoría propuesta, el promedio general arrojo un 2,88 de 5,0 posibles y unas brechas 2,12 que de acuerdo con la clasificación previamente establecida corresponden a una insuficiencia general en el modelo.

Desde los aspectos individuales, las barreras del entorno detectadas estuvieron vinculadas con la competencia y otras opciones que tienen los clientes, sin embargo, la organización no cuenta con estudios o pruebas de referencia para comprender cómo los competidores compensan el impacto negativo de las barreras creadas por ambas partes en el entorno laboral. El apartado del concepto logístico es de las pocas variables que logro la calificación de bueno, debido a que se cuenta al interior de la organización la comprensión del sistema logístico en sí, los sistemas de gestión integrados y las herramientas de contabilidad de costos. Cada uno de estos conceptos es esencial para mejorar la competitividad y mejorar la logística interna.

Por otra parte, la logística inversa y sus resultados muestran la ejecución del proceso de acuerdo con los métodos tradicionales, causando altos costos y cuellos de botella en las actividades de operación y logística de las áreas funcionales directamente involucradas (como almacenes, compras, producción y servicio al cliente).

La Integración del Supply Chain presento la calificación más baja de todas las variables, esto debido a que la cadena de suministro, no tiene un proceso integrado no puede ejecutarse en función de los procesos de red, y el sistema de información y comunicación no cumple con los requisitos necesarios para el mantenimiento en tiempo real de un flujo de información seguro y eficiente, lo que puede aportar un valor agregado al proceso en ejecución.

Por parte de las medidas de desempeño logístico, esta tiene una insuficiencia en la medida que cada área funcional tiene su propio sistema de indicadores que se manejan de manera independiente, y esta información se usa y analiza en cada área, y el grupo de trabajo interfuncional no se usa para explicar y crear un sistema métrico común, por lo tanto cada área se encuentra por su lado sin ningún tipo de coordinación interfuncional.

Por parte de la Tecnología de Organización y Gestión Logística (Supply Chain), este aspecto frente a los cambios e innovaciones en conceptos y tendencias de logística avanzada a nivel mundial muestra un desempeño con poca motivación respecto a este, reduciendo su capacidad de respuesta y causando insatisfacción 
interna y externa del cliente, lo que representa el fracaso de la ejecución del proceso logístico y empeora aún más la estructura logística.

Por parte del Talento Humano la buena calificación en la tecnología es evidente puesto que es el valor más alto requerido por el modelo y está más cerca del valor aproximado requerido. También se debe enfatizar que la capacitación del personal es esencial para el desarrollo profesional y de la compañía. No importa qué actividades económicas se realicen, esto producirá una ventaja competitiva en el mercado.

En la tecnología de almacenaje, los procesos no están mecanizados, lo que implica que las condiciones físicas y ambientales del almacén no aseguran el almacenamiento adecuado de alimentos y materiales en general ni buenas condiciones de higiene y seguridad. Sin embargo, no existe un control automático basado en el modelo de gestión de todas las materias primas, materiales, repuestos, productos intermedios e inventario de productos terminados, pero aún mantiene una programación relativamente efectiva.

Cabe aclarar que al observar el rol de la gestión de la información como generadora de ventaja competitiva para las empresas ya que permite mantener sincronizados los datos hacia los clientes e internamente (Fram \& Licona, 2016), se torna preocupante el hecho que no existe un modelo de captura, procesamiento, transmisión y uso de información basado en tecnología de comunicación computarizada y moderna, si bien existe el ACCOUNT-NET se encuentra basado en un antiguo sistema operativo que no requiere la vinculación o transmisión de información en tiempo real a otros terminales, lo que limita el personal de administración y otros departamentos involucrados en el almacenamiento, la producción y la programación para suministrar sistemas de logística en línea, evitando así decisiones oportunas y correctas. Asimismo, la Tecnología de Software es obsoleta lo que evita sistemas de información y comunicación altamente integrados y en tiempo real, lo que significa que actividades como previsión, planificación, compras, pedidos, inventario, costos, gestión de relaciones con proveedores y usuarios y facturación ya no son compatibles.
En el caso de la manipulación de alimentos es de los pocos aspectos con buena calificación, aunque con posibilidades de mejora en la Operaciones de descarga, manipulación y carga de productos.

En el caso de la tecnología de transporte interno este carece de la aplicación de gestión formal, tampoco tiene un sistema de control y no existe una práctica logística ideal para mejorar la misma como cross-docking, picking y otros métodos relacionados. Por parte del transporte externo, a pesar de obtener una calificación decente, no se considera relevante debido a las características propias de la organización en estudio.

En síntesis, tomando en cuenta los resultados globales e individuales de cada uno de los apartados evaluados por el Modelo Referencial Logístico, se recomienda, en vez de atender cada problemática por individual, proponer un modelo logístico actualizado e integrado que permita poner fin a las ineficiencias detectadas.

\section{Conclusiones y Discusiones.}

Siendo la competitividad uno de los pilares más importantes para el mundo globalizado (Aragón \& Bañón, 2006) no resulta extraño que las organizaciones permitan la elaboración de estudios que posibiliten generar competitividad en su interior. Este es el caso de una organización que al notar varias inconsistencias en su desempeño permitió la una investigación basada en Modelo Referencial Logístico de Donald Bowersox, el cual detecto las problemáticas a nivel especifico y permitió cuantificar las brechas en cada uno de sus trece aspectos. Los resultados demostraron grandes falencias en esos aspectos, por lo tanto, se propone la elaboración de un modelo de gestión logística actualizado que contemple todas las dificultades encontradas, en vez de resolver cada inconveniente. Estos resultados permiten plantear algunas discusiones.

Una de las discusiones más importante que suscitan los resultados del presente artículo recaen en la importancia de las Estrategias diferenciadoras y generadoras de valor (Del Río 
Cortina et al., 2018), en el sentido que el apartado logístico ocupa un lugar central dentro de la cadena productiva y también posibilita mejoras en el desempeño en general. De igual forma, es necesario cambiar el pensamiento respecto al apartado logístico en las organizaciones, dado que se concibe como una estrategia apartada dentro de las áreas funcionales, sin embargo, esta debe ser uno de los aliados principales del marketing (Del Rio Cortina et al., 2017), puesto que permite brindar un mejor servicio, constituyendo una importante herramienta para la competitividad organizacional.

\section{Agradecimientos.}

El presente artículo se construyó a partir de los resultados de la tesis de maestría dirigida Luis Guillermo Garcés Henao y de la autoría de los maestrantes Robinson Carvajal González \& Andrés Del Bosque Vega Quintero de Escuela Naval De Cadetes "Almirante Padilla", Facultad De Administración Naval, Programa Complementación Profesional En Administración Naval En El Año 2019. Adicionalmente se agradece la colaboración del Centro Recreacional De Oficiales Club Naval Santa Cruz De Castillo Grande.

\section{Referencias}

Adarme-Jaimes, W., Arango-Serna, M. D., \& Cogollo-Flórez, J. M. (2012). Medición del desempeño para cadenas de abastecimiento en ambientes de imprecisión usando lógica difusa. Ingenieria y Universidad, 16(1), 95-115.

Aragón Sánchez, A., \& Bañón Rubio, A. (2006). Factores explicativos del éxito competitivo: el caso de las PyMEs del estado de Veracruz. Contaduría y Administración, 216(June), 35-69.

Bowersox, J. ., J.D, C., \& O.K., H. (1986). Logistical Management. A system integration of physical distribution, manufacturing support and materials procurement. (3th editio). Mcmillan Publishing company.

Companys, R., \& Ribas, I. (2015). Some Trends and Applications of Operational Research/Management Science to Operations Management. International Journal of Production Management and Engineering, 3(1), 286-290. https://doi.org/10.4995/ijpme.2015.3459.

Del Rio Cortina, J., Cardona-Arbelaez, D., \& Simancas-Trujillo, R. (2017). Propuesta de un modelo teórico de branding para el posicionamiento de la marca universitaria. Espacios, 38(53).

Del Río Cortina, J., Cardona Arbelaez, D., \& Pérez Olascuaga, S. J. (2018). Estrategias diferenciadoras y generadoras de valor en economías emergentes. Caso. Saber, Ciencia y Libertad, 13(1), 133-144. https://doi.org/10.18041/2382-3240/saber.2018v13n1.2083

Del Río Cortina, J., Velasco Alma Rocio, I., Mendoza Jimenez, W., Pérez Narváez, K., Castilla Puello, C., Orozco Barrera, A., \& Herrera Ballesteros, W. (2009). Una Perspectiva De La Logística Desde La Academia. http://www.eumed.net/libros-gratis/2009c/594/index.htm

Durán, A., González, E., \& Ortiz, M. (2016). Estratégias de marketing e vantagens competitivas em PMEs de móveis. Rede Internacional de Pesquisadores Competitivos, 10(10), 239-253.

Gutiérrez, H. (2010). Qualidade Total e Produtividade (3ra edicio). McGraw-Hill.

Fram, T. O. B., \& Licona, J. M. (2016). La logística y la gestión de la información. Revista Cultural Unilibre, (1), 77-90.

Hernández, H. G., Cardona, D. A., \& Del Rio, J. L. (2017). Direccionamiento estratégico: Proyección de la innovación tecnológica y gestión administrativa en las pequeñas empresas. Informacion Tecnologica, 28(5), 15-22. https://doi.org/10.4067/S0718-07642017000500003

Porter, M. (2015). Estratégia competitiva (6ta edicio). Editorial continental S.A.

Presutti, W. D. (2013). Understanding the Dynamics of the Value Chain. Business Expert Press. 
Prieto, R., Burgos, C., García, J., \& Rincón, Y. (2016). Mercadeo interno para optimizar la calidad de servicio en la banca universal. Revista Venezolana de Gerencia, 21(73), 102-119. https://doi.org/10.31876/revista.v21i73.21059

Rojas De Francisco, L., Bejarano Botero, L. M., \& Marín Valencia, C. F. (2016). Análisis de las estructuras de gestión del servicio en empresas del sector de servicios. AD-Minister, 29, 121-146. https://doi.org/10.17230/ad-minister.29.6

Salas-Navarro, K., Meza, J. A., Obredor-Baldovino, T., \& Mercado-Caruso, N. (2019). Evaluation of the supply chain to improve competitiveness and productivity in the metalworking industry in Barranquilla, Colombia. Informacion Tecnologica, 30(2), 25-32. https://doi.org/10.4067/S0718-07642019000200025 